\title{
TOCA : A HIGHLY USER FRIENDLY APPLICATION PROGRAM FOR THE TUNE, ORBIT, DISPERSION AND CHROMATICITY CORRECTION
}

\author{
F. Iazzourene, Sincrotrone Trieste, Trieste, Italy
}

\begin{abstract}
In order to ease and speed up the intervention of the operators on the machine optics parameters, e.g. the orbit correction, a highly user friendly application program has been developped and fully used from the start of last year (1998). The program allows the acquisition and correction of the closed orbit, the dispersion, the tune and the chromaticity in the storage ring, and the acquisition and correction of the trajectory along the transfer line and the first turn in the storage ring, with a minimum intervention of the operator. The correction strengths are calculated using calculated theoretical optics or measured response matrix. The methods of correction implemented are the SVD method and for the orbit and the trajectory also the MICADO method.
\end{abstract}

\section{INTRODUCTION}

Going towards an increased automation of machine parameter characterization, a highly user friendly multitask application program, TOCA ${ }^{\mathrm{i}}$ (Tune, Orbit, $\underline{\text { Chromaticity }}$ Application program), has been developped[1]. The program is written in $\mathrm{C}$ and the graphical user interface is based on the X11 Motif toolkit. Its main panel is shown in the figure 1. The first vertical window is dedicated to the trajectory along the transfer line and the first turn in the storage ring, and to the orbit and dispersion in the storage ring. The second and the third ones are dedicated to the tune and the chromaticity respectively. Each window has at the bottom three buttons : MEASURE ONLY, MEASURE-CORRECT-MEASURE and UNDO CORRECTION. The first window has also MEASURE and PLOT to get a plot of the selected trajectory or the orbit and/or dispersion. Figure 2 shows the plot of the orbit measured during a shift. The user gets the mean, rms, the min, max and the peak to peak value on the plot. Using the arrows of the keypad, the user can get on the plot the name of the monitor and the position of the beam at the monitor, or the dispersion for a plot of the dispersion. The scales are done by the program. The program performs the requested tasks without any intervention of the user. Furthermore, the button UNDO CORRECTION becomes sensitive only after the user has done a correction. The button , MEASURE-CORRECTMEASURE for the trajectory, orbit and dispersion becomes sensitive only after the user has selected the plane on which he wished to correct, etc... The one go multitask style of the program is not however a complete black box for the user. In the window at the bottom of the main panel, the program writes in step by step every

\footnotetext{
i The new name for the program Orbit-OpticsOptimization.
}

action undertaken and refers in any useful information for the user, for example the results of the correction, the error messages, etc.... The text can be printed by simply clicking in the "print" of the text menu.

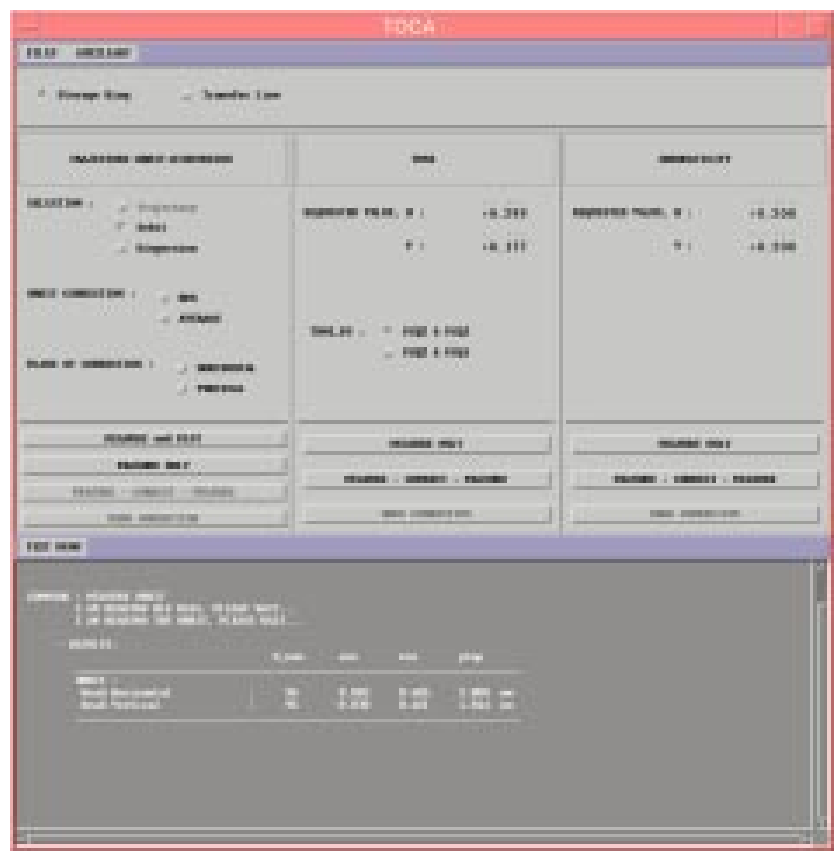

Figure 1: Main panel of the application program TOCA.

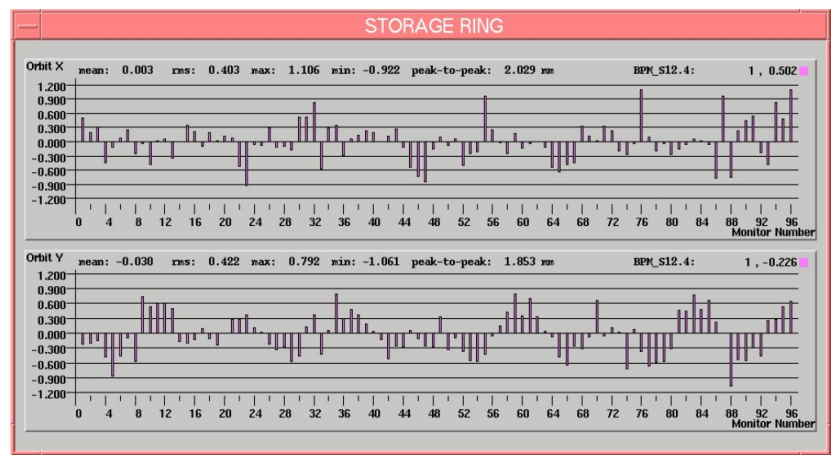

Figure 2: Measured closed orbit during a shift.

To speed up and ease the intervention of the user, all the selections which are more frequent are preselected when the application is started. For example, the chosen system is the storage ring, the families of quadrupole power supplies for the tune correction are PSQ2 and PSQ3, instead of PSQ2 and PSQ1, as the quadrupoles Q3 are better decoupled from the quadrupoles Q2 than the quadrupoles Q1; the requested values for the tune and the chromaticity are preset to the most requested values; the method of correction for the orbit correction is SVD, 
etc.... The data needed by the program is predefined, so that the user can start any task directly. Some of the data are anyway available to the user via two temporary files created each time the user starts the application, so that he can change them as wished. There are two input files, one for the storage ring, the other for the transfer line. A special effort is done to control the data changed by the user to avoid dangerous mishandlings. A temporary output file contains a summary of all the measured parameters: orbit,dispersion,tune, chromaticity and trajectory together with the beam energy and the time of acquisition.

\section{CORRECTION METHODS}

It is well known that the SVD method allows a good rms orbit correction with minimum corrector strengths as compared to the other methods of correction. The chosen method is thus SVD for the orbit and/or dispersion correction in the storage ring, and also for the correction of the beam trajectory along the transfer line. The method is also used for the tune and chromaticity correction for sake of generality. For the orbit and the trajectory, the MICADO method is also available, but we mostly use it to find eventual defective correctors rather than for a correction of the beam orbit or trajectory. By default the method of correction is SVD. The user can choose MICADO method by changing a number 0 to $1,2,3$, etc..., for a MICADO method with $1,2,3$, etc... correctors in the transfer line input file for the beam trajectory correction, or the storage ring input file for the correction of the beam orbit in the storage ring. The spurious dispersion can be corrected with the SVD method. As a correction of the dispersion alone worsens usually the closed orbit, the user is forced to simultaneously correct both the orbit and dispersion, so to select both orbit and dispersion. The orbit correction is weighted via the ratio of the first eigen values found for the orbit and the dispersion separately and a factor preset to 1 . To correct the dispersion alone, the user can set this factor to 0 . As the program is forseen to be used by non physicists, the program sets limits not only on the maximum corrector strengths but also on the additional correctors kicks. The program makes also a severe control on the changed data and sets them to safe values if the user enters values above the maximum allowable. Furthermore, if the number of eigen values chosen by the user lead to kicks or/and additional kicks above the maximum values, the program does not scale the kicks, instead, it decreases the number of eigen values and searches for new correctors strengths up to where the results are within the limits.

\section{RESPONSE MATRIX}

The response matrix can be constructed from the calculated optics, using the well known theoretical response matrices, or measured on the machine. By default, the program creates the response matrix from the calculated optics. To use the measured response matrix, the user simply opens the window found in the menu "auxiliary" and measures the response matrix by clicking in a button "MEASURE RESPONSE MATRIX", if not done yet, then select "Measured response matrix". For the tune, there is also what is called in the program "calculated sensitivity matrix" which constructs the response matrix via changes of the quadrupoles strengths, in theory and not on the ring, and the computation of the changed tunes. It is a calculated response matrix using the change of the tune rather than the specific optics at the quadrupoles. The results of the correction give usually better results than those using the calculated $\beta \mathrm{s}$ at the quadrupoles. For the tune and chromaticity, the efficiency of the measured response matrix as compared to the theoretical one, depends largely on the tune system measurement accuracy and resolution and also on the stability of the beam. For the tune, there is also the compromise between a large enough change of the tune to be able to see the difference together with the care of not crossing some resonance. In this respect, values have been preset which allow a safe tune change during the response matrix measurement, which take into account that the horizontal tune is 0.299 . At Elettra, the use of the response matrix can be helpful when the beam energy is larger than $2 \mathrm{GeV}$ where the quadrupoles and particularly the bending magnets are highly in saturation and the optics are much less known. The response matrix can be measured for the orbit and the dispersion. The efficiency of the measured response matrix depends of course on the BPMs accuracy and resolution, but also on the stability of the beam. The user can hide from the program any monitor or corrector simply by writing in the input file the name of the monitor or the corrector power supply followed by 0 . They can be seen again by the program simply by changing the 0 to 1 or by commenting or deleting the line. This can be done for both the measurement of the orbit and/or dispersion, and for the measurement of the response matrix. The program takes care that the monitors and correctors ignored or found bad during the measurement of the response matrix are also ignored when it calculates the new correctors strengths even if the user might have set the flags differently during the correction or/and the measurement of the orbit or the dispersion. For the transfer line, the input file is created with known defective or taken away monitors with flag 0 .

\section{OTHER TOOLS}

\subsection{Correctors scaling}

The correctors can be scaled. The window is under "auxiliary" menu.

\subsection{Correction with respect to a reference orbit}

The correction of the orbit can be performed with respect to a reference orbit. The user opens the window under 
"auxiliary" menu. He can acquire the reference orbit, plots it, acquire it again, and go back to the previous acquired one. To do the correction with respect to that reference orbit, he just selects "yes" in the window.

\subsection{Tune server}

For the tune measurement, the program accesses the tune server and locks it so that no other user can access it. The program then searches the peaks, reads the tunes, and so on. The tune server is released when the user exits from the application program. To free the server while the application program is running, the user simply clicks in "yes" on the "release tune server" widget which is under the menu "auxiliary".

\subsection{Tune diagram}

The user can view the theoretical tune, the measured tune and a selected working point on a tune diagram by simply clicking in "tune diagram" under the menu "auxiliary". This tool has been provided to get a quick comparison between the theoretical tune and the measured one, to check new tune position, and so on (figure 3 ).

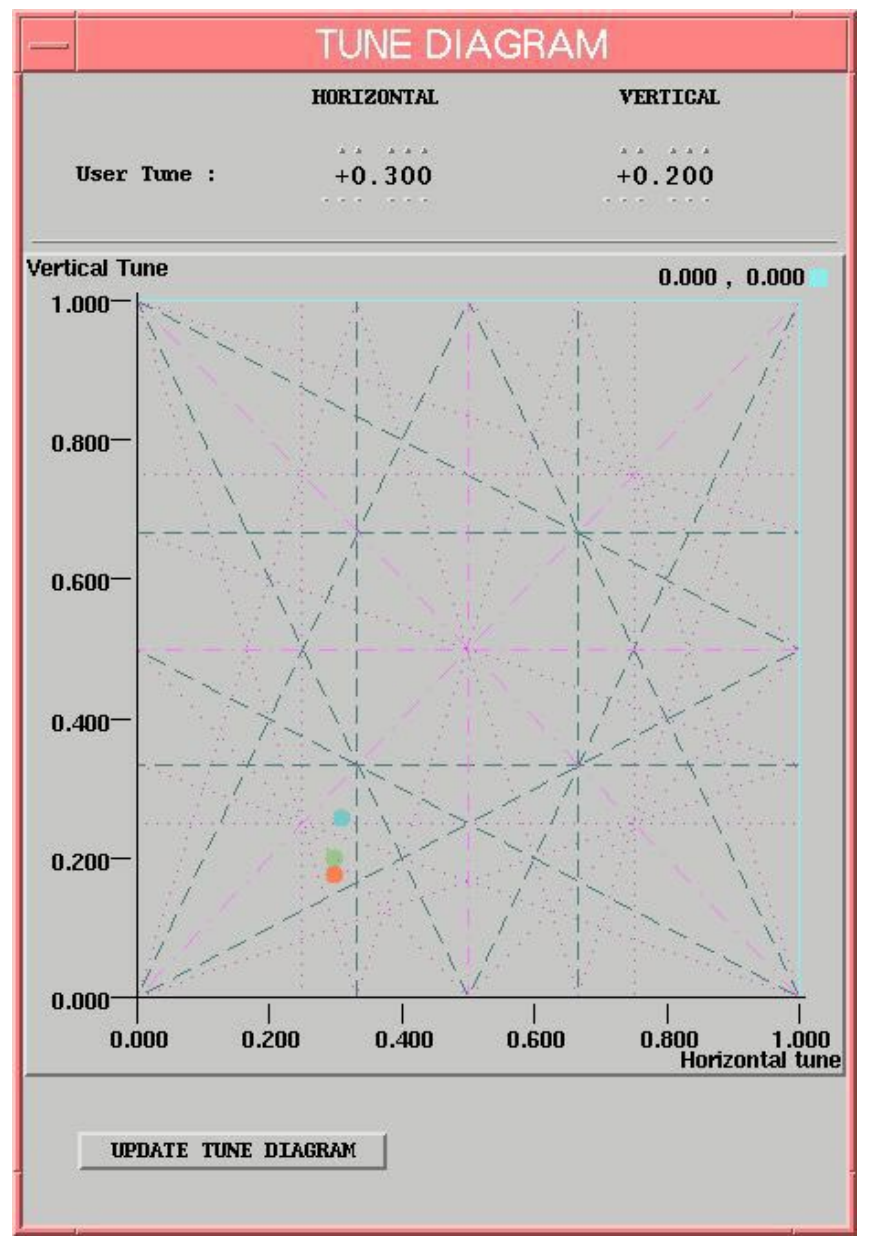

Figure 3: Tune diagram taken during a shift with theoretical tune (blue), measured tune (orange), user tune (green).

\section{ACKNOWLEDGEMENTS}

The author would like to thank the colleagues F. Radovcic and C. Scafuri for their help in the Motif toolkit use.

\section{REFERENCE}

[1] F. Iazzourene, TOCA user's guide, Sincrotrone Trieste (1999). 